\title{
A novel Waikavirus (the family Secoviridae) genome sequence identified in rapeseed (Brassica napus)
}

\author{
D. PARK, Y. HAHN
}

Department of Life Science, Chung-Ang University, Seoul 06974, Republic of Korea

Received September 7, 2018; accepted October 23, 2018

\begin{abstract}
Summary. - The genome sequence of a novel species of the genus Waikavirus (the family Secoviridae), which we named Brassica napus RNA virus 1 (BnRV1), was identified in a rapeseed (Brassica napus) transcriptome dataset. The BnRV1 genome was 12,293 nucleotides long followed by a poly(A) tail. Two open reading frames (ORFs), called ORF1 and ORFX, were predicted. The larger ORF, ORF1, encodes a polyprotein of 3,471 amino acids and the smaller ORF, ORFX, overlaps ORF1 and encodes an 87 aa long protein of unknown function. The BnRV1 ORF1 polyprotein was predicted to undergo proteolytic processing to yield seven mature proteins, including an RNA-dependent RNA polymerase and three distinct coat proteins. The ORF1 and ORFX proteins share sequence similarities with the respective proteins of viruses in the genus Waikavirus, including the bellflower vein chlorosis virus, rice tungro spherical virus, and maize chlorotic dwarf virus. A phylogenetic tree inferred from a conserved segment of the polyproteins of several Secoviridae viruses confirmed that BnRV1 is a novel species of the genus Waikavirus. The BnRV1 genome sequence identified in this study may be useful for the study of waikavirus biology and waikavirus-derived diseases.
\end{abstract}

Keywords: Brassica napus RNA virus 1; Waikavirus; Secoviridae; rapeseed

\section{Introduction}

Viruses of the genus Waikavirus (the family Secoviridae of the order Picornavirales) have a positive-sense singlestranded RNA genome (Sanfaçon et al., 2009; Thompson et al., 2017). There are three known species in the genus Waikavirus: rice tungro spherical virus (RTSV), maize chlorotic dwarf virus (MCDV), and bellflower vein chlorosis virus (BVCV), which are all plant pathogens (Reddick et al., 1997; Sailaja et al., 2013; Seo et al., 2015). RTSV and MCDV are agriculturally important plant pathogens that cause severe production losses in rice and corn, respectively (Reddick et al., 1997; Sailaja et al., 2013).

${ }^{*}$ Corresponding author. E-mail: hahny@cau.ac.kr; phone: +82-2820-5812.

Abbreviations: BnRV1 = Brassica napus RNA virus 1; BVCV = bellflower vein chlorosis virus; $\mathrm{CP}=$ coat protein; $\mathrm{MCDV}=$ maize chlorotic dwarf virus; $\mathrm{NCR}=$ non-coding region; NTP = nucleoside triphosphate-binding protein; $\mathrm{ORF}=$ open reading frame; $\mathrm{RdRp}=\mathrm{RNA}$-dependent RNA polymerase; $\mathrm{RTSV}=$ rice tungro spherical virus
Waikavirus genomes are composed of a mono-segmented single-stranded RNA of about 12 kilobases $(\mathrm{kb})$ with a poly(A) tail, which is encapsulated in an icosahedral particle (Sanfaçon et al., 2009; Thompson et al., 2017). Waikaviruses are known to have at least two well-conserved open reading frames (ORFs) (Firth and Atkins, 2008; Sanfaçon et al., 2009; Thompson et al., 2017). The large ORF, ORF1, encodes a polyprotein that is subsequently processed into seven mature proteins, a hypothetical protein, three distinct coat proteins, a nucleoside triphosphate-binding protein, a 3C-like proteinase, and an RNA-dependent RNA polymerase. The second smaller ORF, ORFX, overlaps ORF1 and encodes an 87 aa protein of unknown function (Firth and Atkins, 2008). Possible additional small ORFs have been proposed near the 3 '-end of the genome of other waikaviruses, although their existence has not been confirmed (Shen et al., 1993; Thole and Hull, 1996; Reddick et al., 1997; Isogai et al., 2000; Verma and Dasgupta, 2007; Sanfaçon et al., 2009).

Rapeseed (Brassica napus), also known as oilseed rape, is a bright-yellow flowering herbaceous plant of the family Brassicaceae (also known as Cruciferae). Rapeseed has 
two subgenomes, one derived from $B$. rapa and the other from $B$. oleracea, indicating that it is a hybrid species that originated from a recent hybridization event (Mason and Snowdon, 2016). Rapeseed is mainly cultivated to produce edible seed oil, and rapeseed oil is a rich source of natural bioactive components, including a range of antioxidants (Szydłowska-Czerniak, 2013).

High-throughput RNA sequencing (RNA-seq) is a powerful, cost-effective tool for RNA virus discovery in plants, as well as viral diagnostics and virus evolution research (Park and Hahn, 2017; Park et al., 2017, 2018; Roossinck, 2017). In this paper, a novel RNA virus genome sequence was identified in a transcriptome dataset obtained from rapeseed tissues (Liu et al., 2017). Sequence comparison and phylogenetic analyses showed that the novel RNA virus is a distinctive species of the genus Waikavirus in the family Secoviridae.

\section{Materials and Methods}

Data collection and sequence assembly. RNA-seq data obtained from the young floral buds of rapeseed plants (Acc. Nos. SRR2052475, SRR2052499, SRR2052502, and SRR2052505) (Liu et al., 2017)were downloaded from the Sequence Read Archive (SRA) of National Center for Biotechnology Information (NCBI). The dataset contains about 19.95 gigabases from 239.7 million reads.

Raw sequence data were trimmed to collect high-quality reads using the Sickle program (version 1.33; https://github.com/najoshi/ sickle). High-quality reads were de novo assembled into contigs using the SPAdes Genome Assembler (version 3.10.1; http://spades. bioinf.spbau.ru) (Bankevich et al., 2012). Each of the four RNA-seq runs was independently assembled.

Viral data collection. A BLAST-searchable database containing representative viral RNA-dependent RNA polymerase (RdRp) motif sequences was prepared from the Pfam database (release 31.0; http://pfam.xfam.org). A total of 394 viral RdRp motif sequences were obtained from 19 families (PF00602, PF00603, PF00604, PF00680, PF00946, PF00972, PF00978, PF00998, PF02123, PF03431, PF04196, PF04197, PF05788, PF05919, PF07925, PF08467, PF08716, PF08717, and PF12426). A BLASTX search was performed against the representative RdRp motif database using the assembled rapeseed transcriptome contigs as queries to identify potential virus-derived sequences.

RNA sequence analysis. The rapeseed RNA-seq reads were mapped to a viral contig using the BWA program (version 0.7.17r1194; https://github.com/lh3/bwa) (Li and Durbin, 2009) to collect virus-derived reads and to examine sequencing depth. The SAMtools and BCFtools programs (version 1.6; http://www. htslib.org) (Li et al., 2009) were used to identify single nucleotide polymorphisms (SNPs). Integrative Genomics Viewer was used to visualize sequencing coverage and depth (Robinson et al., 2011).
ORF and protein characterization. The getorf program (version 6.6.0.0; http://www.bioinformatics.nl/cgi-bin/emboss/getorf) of the EMBOSS package was used to predict ORFs and their encoded protein sequences. Sequence similarity searches of the putative viral contig against all known nucleotide and protein sequences were conducted using the BLAST programs at the NCBI website (https:// blast.ncbi.nlm.nih.gov/Blast.cgi). The Pfam database (release 31.0; http://pfam.xfam.org) was utilized to characterize the functional domains in the viral genome.

Phylogenetic analysis. Multiple sequence alignments were generated using the MUSCLE program (version 3.8.31; https://www. drive5.com/muscle) (Edgar, 2004). A phylogenetic tree was inferred by the neighbor-joining method using MEGA (version X; https:// www.megasoftware.net) (Kumar et al., 2018).

\section{Results and Discussion}

One of the contig sequences assembled from the rapeseed (Brassica napus) flower bud RNA-seq set SRR2052475 contains a putative protein coding region showing high similarity with an RdRp motif (Pfam Acc. No. PF00680) of the RTSV ORF1 polyprotein (UniProt Acc. No. Q83034). RTSV is the prototype species of the genus Waikavirus of the family Secoviridae (Shen et al., 1993). The contig was thought to be a genome sequence derived from a novel RNA virus related to the genus Waikavirus and was named Brassica napus RNA virus 1 (BnRV1). The BnRV1 genome sequence with annotation information has been deposited in the NCBI nucleotide database under Acc. No. MH844554.

The BnRV1 genome contig, which was assembled from 58,986 RNA-seq reads, is 12,293 nucleotides (nt) long followed by a poly (A) tail. A single nt sequence variation ( $\mathrm{G}$ or $\mathrm{T}$ ) was found at position 9660 , which resulted in a methionine/isoleucine amino acid (aa) polymorphism in the ORF1 polyprotein. This indicated that the assembled BnRV1 genome was derived from a highly homogeneous population and may be descendant of a single clone.

Sequence comparison and ORF prediction revealed two well-conserved ORFs in the BnRV1 genome (Fig. 1), the larger ORF1 and the overlapping smaller ORFX, which were 10,416 nt (position 631-11046) and $264 \mathrm{nt}$ (position 923-1186) long, respectively. The 5 ' non-coding region (NCR) was $630 \mathrm{nt}$ long, and the 3' NCR was 1,247 nt long and was followed by a poly(A) tail. None of the other small ORFs previously suggested in other viruses of the genus Waikavirus were found (Shen et al., 1993; Reddick et al., 1997; Sanfaçon et al., 2009).

BnRV1 ORF1 encoded a polyprotein of 3,471 aa. Pfam analysis showed that the polyprotein contained five conserved domains: a waikavirus capsid protein 1 (Pfam Acc. No. PF12264; aa positions 711-907), picornavirus capsid protein (PF00073; 895-1061), RNA helicase (PF00910; 1800-1906), 


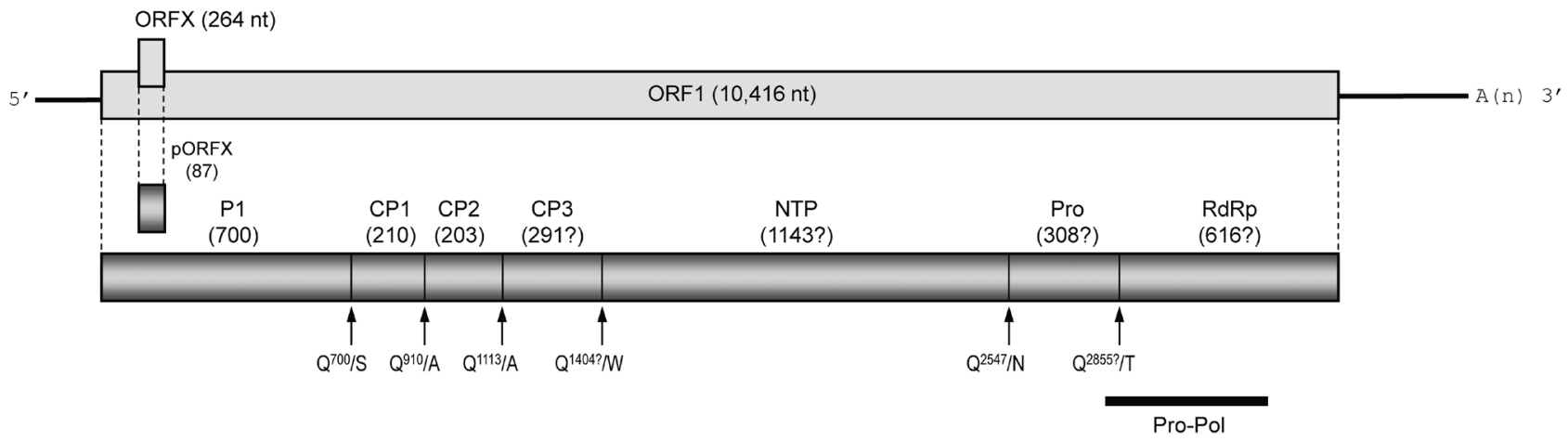

Fig. 1

Schematic representation of the BnRV1 genome

The BnRV1 genome contains two ORFs: the larger ORF1 (10,416 nt) and the overlapping smaller ORFX (264 nt), which are marked as boxes (top). ORF1 encodes a polyprotein (3,417 aa) that undergoes proteolytic cleavage to generate a hypothetical protein ( $\mathrm{P} 1)$, three coat proteins (CP1, CP2, and CP3), a nucleoside triphosphate-binding protein (NTP), a 3C-like proteinase (Pro), and an RdRp (middle). ORFX produces a small protein (pORFX, 87 aa) of unknown function. The aa residues and positions of the putative cleavage sites are marked by arrows below the polyprotein. The seven mature proteins are indicated with their respective sizes. Poorly supported sizes or positions are indicated by question marks. The "Pro-Pol" segment, which was used for the phylogenetic analysis, is marked with a line (bottom).

tungro spherical virus-type peptidase (PF12381;2634-2864), and RdRp (PF00680; 2903-3413).

A BLASTP search was performed against the NCBI nonredundant protein database using the BnRV1 polyprotein sequence as a query. The BnRV1 polyprotein showed strong sequence similarity with the polyproteins of BVCV (Seo et al., 2015), RTSV, and MCDV (Reddick et al., 1997). These three viruses are species of the genus Waikavirus. The BnRV1 polyprotein also showed significant sequence similarity with the polyproteins of carrot necrotic dieback virus (Menzel and Vetten, 2008) and parsnip yellow fleck virus (Turnbull-Ross et al., 1992). These two viruses are members of the genus Sequivirus, a sister taxon of the genus Waikavirus.

The virus with the highest similarity to BnRV1 was BVCV, and the ORF1 polyproteins showed 51\% sequence identity, with almost full coverage. A BLASTN search against known viral genomes using the BnRV1 genome sequence as a query also revealed that BVCV was the closest to BnRV1, with $65 \%$ nt sequence identity and $44 \%$ coverage. Strong sequence similarity among the polyproteins of BnRV1 and other waikaviruses, including BVCV, RTSV, and MCDV, was observed in the multiple sequence alignment (Supplementary Fig. S1), indicating that BnRV1 is a novel member of the genus Waikavirus.

The BnRV1 polyprotein is predicted to be cleaved into seven mature proteins: a hypothetical protein $(\mathrm{P} 1)$, three distinct coat proteins (CP1, CP2, and $\mathrm{CP} 3)$, a nucleoside triphosphate-binding protein (NTP), a 3C-like proteinase (Pro), and an RdRp. The consensus sequence for cleavage sites is " $Q / X$," where " $Q$ " is a glutamine residue and " $\mathrm{X}$ " is any aa residue (Shen et al., 1993; Reddick et al., 1997; Sanfaçon et al., 2009; Seo et al., 2015). Putative cleavage sites in the

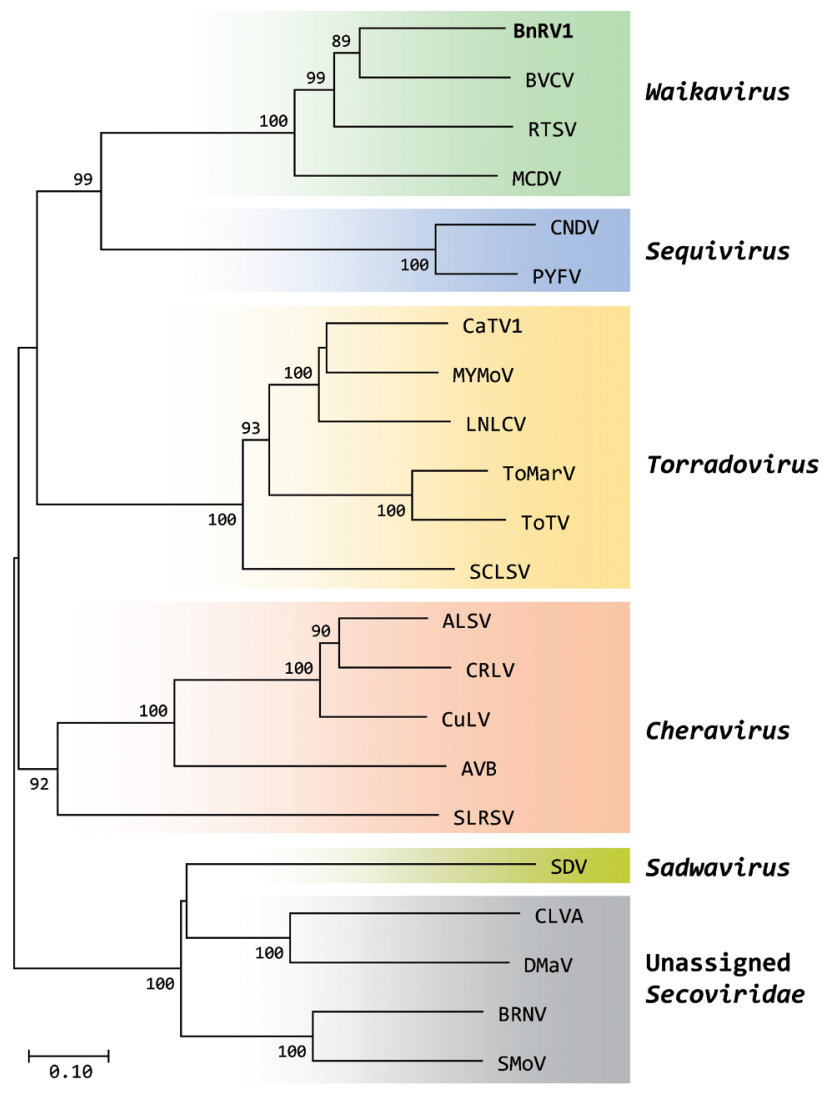

Fig. 2

Phylogenetic tree of BnRV1 and other Secoviridae viruses A phylogenetic tree was inferred based on the "Pro-Pol" segment sequences of BnRV1 and other representatives of Secoviridae viruses using the neighbor-joining method. The bootstrap percentages calculated from 1,000 replicates are shown at the nodes (bootstrap support values of $80 \%$ or less are not marked). See Table 1 for the full names and Acc. Nos. of the viruses. 
BnRV1 polyprotein were predicted based on comparison to the reported cleavage sites of other waikavirus polyproteins (Fig. 1). Examination of a multiple sequence alignment of waikavirus polyproteins revealed that the cleavage sites for $\mathrm{P} 1 / \mathrm{CP} 1, \mathrm{CP} 1 / \mathrm{CP} 2, \mathrm{CP} 2 / \mathrm{CP} 3$, and NTP/Pro were well aligned (Supplementary Fig. S1). For these four sites, the aligned glutamine residues of the BnRV1 polyprotein were predicted to be cleavage sites. In contrast, the sites for CP3/NTP and Pro/RdRp were poorly aligned. For these two sites, nearby glutamine residues were chosen as putative cleavage sites. The six predicted cleavage sites of the BnRV1 polyprotein are as follows (the positions of the glutamine residues are given in parentheses): $\mathrm{Q}(700) / \mathrm{S}$ for $\mathrm{P} 1 / \mathrm{CP} 1, \mathrm{Q}(910) / \mathrm{A}$ for CP1/CP2, Q(1113)/A for CP2/CP3, Q(1404)/W for CP3/ NTP, Q(2547)/N for NTP/Pro, and Q(2855)/T for Pro/RdRp.

A conserved segment of the polyprotein, which is known as the "Pro-Pol" region, has been used as a taxonomical indicator for viruses of the order Picornavirales, including the genus Waikavirus (Sanfaçon et al., 2009; Seo et al., 2015; Thompson et al., 2017). The "Pro-Pol" segment spans from the "CG" motif of the Pro to the "GDD" motif of the RdRp (aa positions 2815-3269 in the BnRV1 polyprotein). Comparison of the BnRV1 "Pro-Pol" sequence with those of representative Secoviridae viruses revealed a close relationship (Table 1). The BnRV1 "Pro-Pol" sequence showed 29-67\% identity with those of known Secoviridae. Among them, three Waikavirus species - BVCV, RTSV, and MCDV - showed $67 \%, 61 \%$, and $57 \%$ identity, respectively. One of the criteria for species demarcation within the family Secoviridae by International Committee on Taxonomy of Viruses is that the "Pro-Pol" sequence shows less than $80 \%$ identity (http://www.ictv.global/report/secoviridae) (Thompson et al., 2017). According to this criterion, BnRV1 is considered to be a novel species in the genus Waikavirus.

A multiple sequence alignment of the collected "Pro-Pol" sequences of representative Secoviridae viruses and BnRV1 was generated (Supplementary Fig. S2). A phylogenetic tree based on this alignment confirmed the classification of BnRV1 in the genus Waikavirus (Fig. 2).

The BnRV1 genome was predicted to have a second ORF, called ORFX, that overlaps the N-terminal region of the ORF1 polyprotein and encodes an 87 aa protein (Fig. 1). The BnRV1 ORFX protein sequence showed high sequence similarity with other waikavirus ORFX proteins (Supplementary Fig. S3). Interestingly, the sequence conservation for the ORFX proteins was higher than that for the overlapping regions of the ORF1 polyproteins. For example, the ORFX proteins of BnRV1 and BVCV showed $74 \%$ aa sequence identity, while the overlapping ORF1 polyprotein regions showed 53\% identity. The ORFX proteins have only been found in the four Waikavirus species. No homologous proteins have been found in other viruses or other cellular

Table 1. Sequence comparison of the "Pro-Pol" segments of BnRV1 and representatives of Secoviridae viruses

\begin{tabular}{|c|c|c|c|c|}
\hline Genus & Virus & Acronym & NCBI Acc. No. & Sequence identity ${ }^{\mathrm{a}}$ \\
\hline \multirow[t]{3}{*}{ Waikavirus } & bellflower vein chlorosis virus & BVCV & NC_027915 & $306 / 455(67 \%)$ \\
\hline & rice tungro spherical virus & RTSV & NC_001632 & $282 / 463(61 \%)$ \\
\hline & maize chlorotic dwarf virus & MCDV & NC_003626 & $262 / 458(57 \%)$ \\
\hline \multirow[t]{2}{*}{ Sequivirus } & carrot necrotic dieback virus & CNDV & EU980442 & $166 / 487(34 \%)$ \\
\hline & parsnip yellow fleck virus & PYFV & NC_003628 & $161 / 488(33 \%)$ \\
\hline \multirow[t]{6}{*}{ Torradovirus } & carrot torradovirus 1 & CaTV1 & NC_025479 & $149 / 460(32 \%)$ \\
\hline & motherwort yellow mottle virus & MYMoV & NC_035218 & $142 / 462(31 \%)$ \\
\hline & lettuce necrotic leaf curl virus & LNLCV & NC_035214 & $147 / 462(32 \%)$ \\
\hline & tomato marchitez virus & ToMarV & NC_010987 & $121 / 385(31 \%)$ \\
\hline & tomato torrado virus & ToTV & NC_009013 & $120 / 401(30 \%)$ \\
\hline & squash chlorotic leaf spot virus & SCLSV & NC_035221 & $144 / 476(30 \%)$ \\
\hline \multirow[t]{5}{*}{ Cheravirus } & apple latent spherical virus & ALSV & NC_003787 & $144 / 476(30 \%)$ \\
\hline & cherry rasp leaf virus & CRLV & NC_006271 & $141 / 457(31 \%)$ \\
\hline & currant latent virus & $\mathrm{CuLV}$ & NC_029038 & $140 / 457(31 \%)$ \\
\hline & Arracacha virus B & AVB & NC_020898 & $144 / 487(30 \%)$ \\
\hline & strawberry latent ringspot virus & SLRSV & NC_006964 & $114 / 386(30 \%)$ \\
\hline Sadwavirus & satsuma dwarf virus & SDV & NC_003785 & $111 / 371(30 \%)$ \\
\hline \multirow{4}{*}{$\begin{array}{l}\text { Unassigned } \\
\text { Secoviridae }\end{array}$} & chocolate lily virus A & CLVA & NC_016443 & $116 / 374(31 \%)$ \\
\hline & Dioscorea mosaic associated virus & $\mathrm{DMaV}$ & NC_031766 & $137 / 473(29 \%)$ \\
\hline & black raspberry necrosis virus & BRNV & NC_008182 & $141 / 470(30 \%)$ \\
\hline & strawberry mottle virus & $\mathrm{SMoV}$ & NC_003445 & $140 / 472(30 \%)$ \\
\hline
\end{tabular}

amino acid sequence identities are reported as "identical residues/aligned length (\% identity)." 
organisms. The strong conservation of the ORFX protein sequences suggests that they may play an important role in the biology of waikaviruses.

In conclusion, a genome sequence of a novel RNA virus, BnRV1, was identified in rapeseed. Analysis of the genomic features and phylogeny indicated that BnRV1 is a novel fourth member of the genus Waikavirus in the family $\mathrm{Se}$ coviridae. Three plant viruses are known to infect rapeseed - Brassica yellow virus, turnip crinkle virus, and turnip yellow virus (retrieved from the Virus-Host database at https:// www.genome.jp/virushostdb) (Mihara et al., 2016). BnRV1 is the fourth virus that is associated with rapeseed. Considering the economic value of rapeseed and the potential pathogenicity of waikaviruses, the BnRV1 genome sequence reported in this study may be useful for the study of waikavirus biology and the control of waikavirus-derived diseases.

Acknowledgment. This research was supported by the National Research Foundation of Korea funded by the Government of Korea [grant Nos. NRF-2017R1A1B4005866 and NRF2018R1A5A1025077].

Supplementary information is available in the online version of the paper.

\section{References}

Bankevich A, Nurk S, Antipov D, Gurevich AA, Dvorkin M, Kulikov AS, Lesin VM, Nikolenko SI, Pham S, Prjibelski AD, Pyshkin AV, Sirotkin AV, Vyahhi N, Tesler G, Alekseyev MA, Pevzner PA (2012): SPAdes: a new genome assembly algorithm and its applications to single-cell sequencing. J. Comput. Biol. 19, 455-477. https://doi.org/10.1089/ cmb.2012.0021

Edgar RC (2004): MUSCLE: multiple sequence alignment with high accuracy and high throughput. Nucleic Acids Res. 32, 1792-1797. https://doi.org/10.1093/nar/gkh340

Firth AE, Atkins JF (2008): Bioinformatic analysis suggests that a conserved ORF in the waikaviruses encodes an overlapping gene. Arch. Virol. 153, 1379-1383. https://doi. org/10.1007/s00705-008-0119-5

Isogai M, Cabauatan PQ, Masuta C, Uyeda I, Azzam O (2000): Complete nucleotide sequence of the rice tungro spherical virus genome of the highly virulent strain Vt6. Virus Genes 20, 79-85. https://doi.org/10.1023/A:1008116408733

Kumar S, Stecher G, Li M, Knyaz C, Tamura K (2018): MEGA X: Molecular Evolutionary Genetics Analysis across computing platforms. Mol. Biol. Evol. 35, 1547-1549. https://doi. org $/ 10.1093 / \mathrm{molbev} / \mathrm{msy} 096$

Li H, Durbin R (2009): Fast and accurate short read alignment with Burrows-Wheeler transform. Bioinformatics 25, 17541760. https://doi.org/10.1093/bioinformatics/btp324

Li H, Handsaker B, Wysoker A, Fennell T, Ruan J, Homer N, Marth G, Abecasis G, Durbin R, 1000 Genome Project Data Processing Subgroup (2009): The Sequence Alignment/Map format and SAMtools. Bioinformatics 25, 2078-2079. https://doi.org/10.1093/bioinformatics/btp352

Liu XQ, Yu CY, Dong JG, Xu AX, Hu SW (2017): De novo transcriptome reconstruction of a thermo-sensitive male sterility mutant in rapeseed (Brassica napus; Brassicaceae). Appl. Plant Sci. 5, apps.1700077. https://doi.org/10.3732/ apps. 1700077

Mason AS, Snowdon RJ (2016): Oilseed rape: learning about ancient and recent polyploid evolution from a recent crop species. Plant Biol. (Stuttg) 18, 883-892. https://doi.org/10.1111/ plb.12462

Menzel W, Vetten HJ (2008): Complete nucleotide sequence of an isolate of the Anthriscus strain of parsnip yellow fleck virus. Arch. Virol. 153, 2173-2175. https://doi.org/10.1007/ s00705-008-0240-5

Mihara T, Nishimura Y, Shimizu Y, Nishiyama H, Yoshikawa G, Uehara H, Hingamp P, Goto S, Ogata H (2016): Linking virus genomes with host taxonomy. Viruses 8,66 . https:// doi.org/10.3390/v8030066

Park D, Goh CJ, Kim H, Hahn Y (2018): Identification of two novel amalgaviruses in the common eelgrass (Zostera marina) and in silico analysis of the amalgavirus +1 programmed ribosomal frameshifting sites. Plant Pathol. J. 34, 150-156.

Park D, Hahn Y (2017): Genome sequence of spinach cryptic virus 1 , a new member of the genus Alphapartitivirus (family Partitiviridae), identified in spinach. J. Microbiol. Biotechnol. 27, 834-837. https://doi.org/10.4014/jmb.1611.11026

Park D, Kim H, Hahn Y (2017): Genome sequence of a distinct watermelon mosaic virus identified from ginseng (Panax ginseng) transcriptome. Acta Virol. 61, 479-482. https:// doi.org/10.4149/av $2017 \quad 410$

Reddick BB, Habera LF, Law MD (1997): Nucleotide sequence and taxonomy of maize chlorotic dwarf virus within the family Sequiviridae. J. Gen. Virol. 78, 1165-1174. https:// doi.org/10.1099/0022-1317-78-5-1165

Robinson JT, Thorvaldsdottir H, Winckler W, Guttman M, Lander ES, Getz G, Mesirov JP (2011): Integrative genomics viewer. Nat. Biotechnol. 29, 24-26. https://doi.org/10.1038/ nbt. 1754

Roossinck MJ (2017): Deep sequencing for discovery and evolutionary analysis of plant viruses. Virus Res. 239, 82-86. https://doi.org/10.1016/j.virusres.2016.11.019

Sailaja B, Anjum N, Patil YK, Agarwal S, Malathi P, Krishnaveni D, Balachandran SM, Viraktamath BC, Mangrauthia SK (2013): The complete genome sequence of a south Indian isolate of rice tungro spherical virus reveals evidence of genetic recombination between distinct isolates. Virus Genes 47, 515-523. https://doi.org/10.1007/s11262$\underline{013-0964-5}$

Sanfaçon H, Wellink J, Le Gall O, Karasev A, van der Vlugt R, Wetzel T (2009): Secoviridae: a proposed family of plant viruses within the order Picornavirales that combines the families Sequiviridae and Comoviridae, the unassigned genera Cheravirus and Sadwavirus, and the proposed genus Torradovirus. Arch. Virol. 154, 899-907. https:// doi.org/10.1007/s00705-009-0367-z

Seo JK, Kwak HR, Lee YJ, Kim J, Kim MK, Kim CS, Choi HS (2015): Complete genome sequence of bellflower vein chlorosis 
virus, a novel putative member of the genus Waikavirus. Arch. Virol. 160, 3139-3142. https://doi.org/10.1007/ s00705-015-2606-9

Shen P, Kaniewska M, Smith C, Beachy RN (1993): Nucleotide sequence and genomic organization of rice tungro spherical virus. Virology 193, 621-630. https://doi.org/10.1006/ viro.1993.1170

Szydłowska-Czerniak A (2013): Rapeseed and its products-sources of bioactive compounds: a review of their characteristics and analysis. Crit. Rev. Food Sci. Nutr. 53, 307-330. https://doi.org/10.1080/10408398.2010.529959

Thole V, Hull R (1996): Rice tungro spherical virus: Nucleotide sequence of the 3 ' genomic half and studies on the two small 3' open reading frames. Virus Genes 13, 239-246. https://doi.org/10.1007/BF00366984
Thompson JR, Dasgupta I, Fuchs M, Iwanami T, Karasev AV, Petrzik K, Sanfacon H, Tzanetakis I, van der Vlugt R, Wetzel T, Yoshikawa N, ICTV Report Consortium (2017): ICTV virus taxonomy profile: Secoviridae. J. Gen. Virol. 98, 529-531. https://doi.org/10.1099/igv.0.000779

Turnbull-Ross AD, Reavy B, Mayo MA, Murant AF (1992): The nucleotide sequence of parsnip yellow fleck virus: a plant picorna-like virus. J. Gen. Virol. 73 ( Pt 12), 3203-3211. https://doi.org/10.1099/0022-1317-73-12-3203

Verma V, Dasgupta I (2007): Sequence analysis of the complete genomes of two rice tungro spherical virus isolates from India. Arch. Virol. 152, 645-648. https://doi.org/10.1007/ s00705-006-0861-5 


\title{
Supplementary information
}

\section{A novel Waikavirus (the family Secoviridae) genome sequence identified in rapeseed (Brassica napus)}

\author{
D. PARK, Y. HAHN ${ }^{\star}$
}

Department of Life Science, Chung-Ang University, Seoul 06974, Republic of Korea

Received September 7, 2018; accepted October 23, 2018

Fig. S1. Multiple sequence alignment of the ORF1 polyprotein sequences of BnRV1 and other waikaviruses

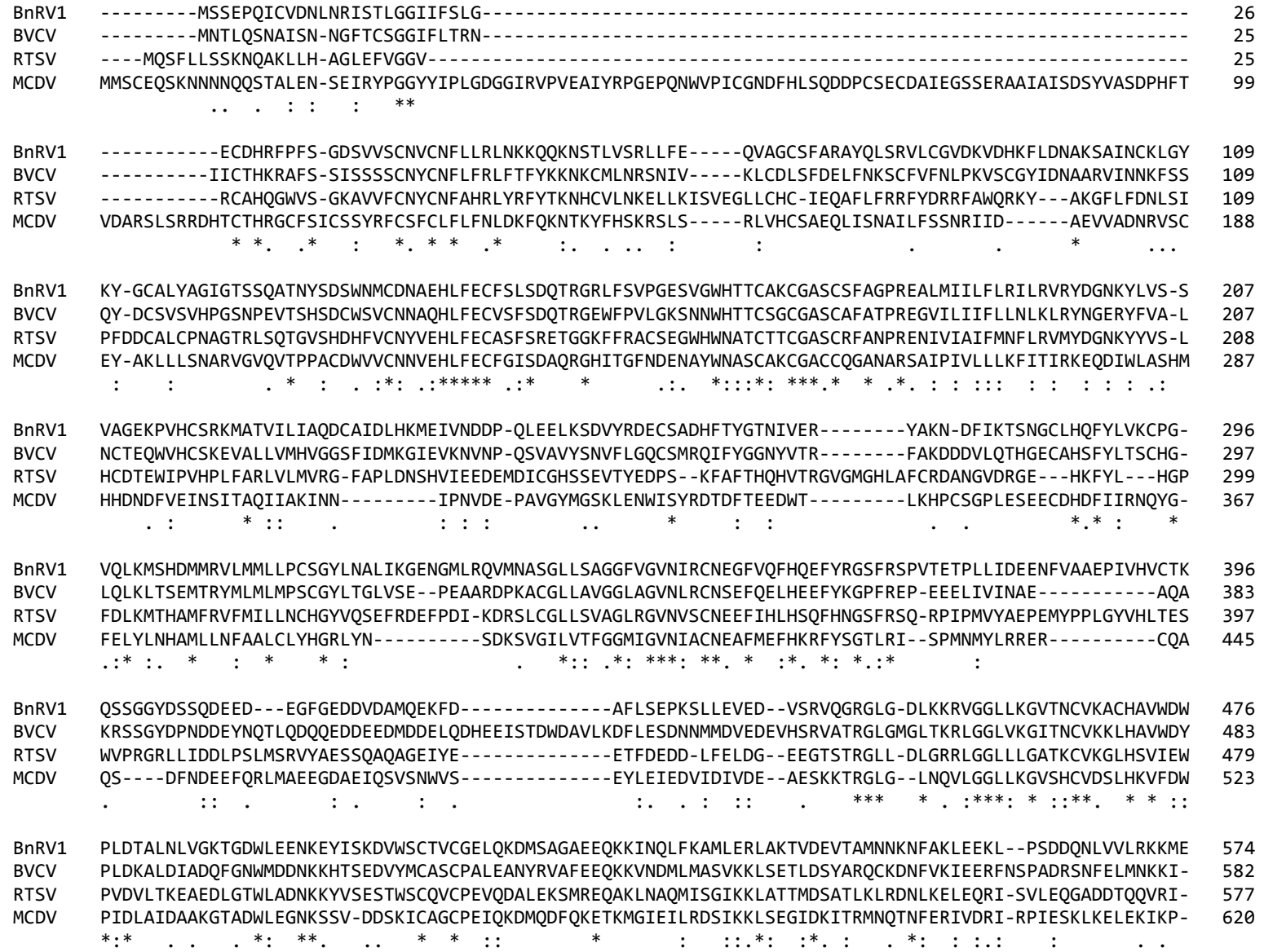


BnRV1 LMQDELTRHAGDIHAVKTEVERMDSNLGPFNGEACILSIAEIKVDMKEMKERLNQLEREPKGSKLPTSIPPPRDRPRPTPTSSDAGEQLPIPRMSVMDGS BVCV - MCDV -

NVRQPPPGMSKSRQARWFEKQMLMRQSGSTEFVGTEMQAEVVESSRVVARGSTEEHNPFLSKLYLGSVSWSVSDGEGTVLQDFDIPSAVWGSNSRLADIL BVCV KIGRPSNPISLPQPIRKFGISA-TKQSGD RTSV --RRRPPVVEMSEA------QAGETVIVGGDEEQEAHQDSSVAAAGPADEHNAMLQKIYLGSFKWKVSDGGGSILKTFSLPSDIWAANDRMKNFL MCDV KIRVKAKRVEK-...-.

SYFQYYTVDGMDFYVTLTSIGMQGGTLMLVWDALSCATRQKTASVFQLSNLPKAFIHASESAEQVFSIDSPSIQHQMCTSGSEGSLGNLGTLKICIANVL BVCV SCFQYYTCDGLRFHVTTTSVGMQGGTLMLSWDAMSCATKQKIDSVLQLSNLPSAYIGASDSVNQVFEVSSPSIQHIMCTSGSEGSIGGLGTLKISVANVL RTSV SYFQYYTCEGMTFTLTITSIGLHGGTLLVAWDALSSATRRGIVSMIQLSNLPSMTLHASGSSIGTLTVTSPAIQHQICTSGSEGSLANLGSLVISVANVL MCDV SYFQYYKATGLTFRISTTCIPMHGGTLFAAWDACGCATRQGIATAVQLTGLPGIMIEAHSSSLTTFSVEDPLTQSTVCLSGSEHSFGRIGILKICCLNVL

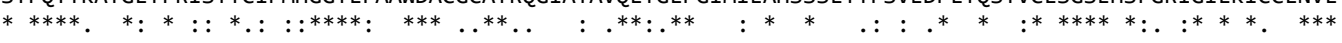

NASSETSQSASVNVWLKFRNPRMSLYTRKHELVMSOACMDVEETHGLESCEAMIATGKWSTTSSTNLMELTVHPTACHLERGMLSQTSLSVIASMFGRWR BVCV NASTDTSQKVQVNMWVSFINPKLSFVTVPHIPLVSOSSATLSNLAGIESFESIVAADKWSTTSPTNLLELTVHPTACHVKDGLVTQTSLSVVSSLFNRWR RTSV CADSASAQELNVNAWVQFNKPKLSYWTAQHSIA--QSG-GFEESQDLGDLQAIIATGKWSTTSDKNLMEIIVHPTACYVSEKLIYQTNLSVVAHMFAKWS MCDV NAPQAATQSVSVNVWVKFDGVKFHFYSLKKQPVVSQM--LVDKLTNLGEMGCVVATGTWSTTSSLNLLQLNVHPTACFISDGLVTQTPLSVIAHAFARWR

BnRV1 BVCV

RTSV MCDV

BnRV1

BVCV

RTSV

MCDV

BnRV1

BVCV

RTSV

MCDV

BnRV1

BVCV

RTSV

MCDV

BnRV1

BVCV

RTSV

MCDV

BnRV1

BVCV

RTSV

MCDV

BnRV1

BVCV

RTSV

MCDV

BnRV1

BVCV

RTSV

MCDV

BnRV1

BVCV

RTSV

MCDV
GSLRFKFVFGASMFVKGKVAVSAIPIAFRKEKLSVAQLLSFPSVICDLNTANKEFIFDVPYNSIGEDSYVTRDSLYDMSCYNGDFVVSRLHMVVLDPLVM 1074 GSIKYRIIFGASMFVKGKMAVSAVPVVFRNRKMSVEEICAFPCLICDLSSQNREFTFEVPYVSIGTDSYVVRDALYDTSSYSAKFVVSRLHFVVLDPLVM 1046 GSMRYTFVFGASMFDRGKIMVSAVPVOFRNSKLTLSOMAAFPSMVCDLSMETREFTFEVPYISIGKMSLVCKDYLFDISSYNADLVVSRLHVMILDPLVK 1015 GSLKFTITFGASMFTRGRVLVAAIPVAKRKETLTIEEISGYHNVMCLLNGERTSFELEVPYHSVGEDSYVCRDALFDVSSYAQNFMITRLHMVVIDTLVM 1069

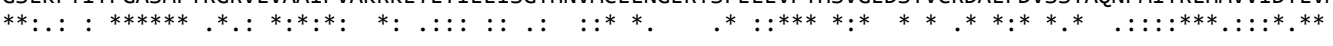

NANASNSISFFVMMGPGDDFTLSOLLGVKSEYVNRNLK-OA-FGRSLNSGGLNGDGFSRWCGVPSVLAKFELNADKKN- - - - - ALHFMVSPYYRRFPPC 1166 NANASNSVSFIVTQSPGKDFQLSQLSGVKAEFVDRRLKPQG-FGRTLTCNGLMGGGFNDWCEIPSVLWKFTLDAGLKN-_-.-ALHLMVAPSYRSMPPC 1139 TGNASNSIGFYVVAGPGKGFKLHQMCGVKSQFAHDVLTAQD-FGRSLSCSRLLGNGFKEWCSRESLLMRVPLKSGKKR--- - - AFKYAVTPRMRTLPPE 1108 SSNASNTISYCVMMGPGKDLELRYLNGVHAQRNVRELKAQVSLGFSLQSGRNIGVGFSDLLKRWAHLLTLHFDENNEKSEEKVGSYIVTVAPSYRAFPQH 1169

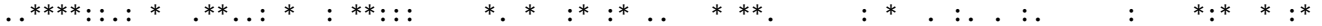

VTSLSWLSQIFVEWRGSLQYTLRAHSQDRAKGSLVRIWHDANGSTISREEFEFLSDVDPPSGMRVLYWNPVLSDTYTFTVPYRARTKKLIIMKARYEPHE 1266 TTSLSWLAQLFVEWSGSLVYTLRAHSQAKQITSFVRIWYDSNGSTQSENESEFLSNVDPPAGVKVHYWRPGEQDKIVITVPFCARTPKLLLPKSRYEPTV 1239 ATSLSWLSOIFVEWRGSLTYTIHVOSGSAIOHSYMRIWYDPNGKTDEK -EVKFLDSAHPPAGIKVYHWDLKIGDSFRFTVPYCARTEKLQIPKA-YASTP 1206 NTLLSWFSQLFVQWQGSLCYRLHVDSQERRYGGYLRIWHDPNGSLDEGVEFAMSTNLEPPPGAFVKYWNYNEQSEFEFVVPYTARTPRLFVPKAMIPTDS 1269

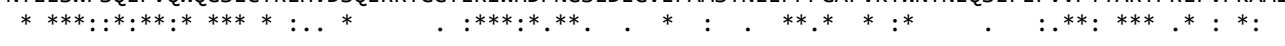

YDWVRCYNGSVVIDFEGPGEMSLELSVSAGSDFEMFEQTVAPRCGNVTKAFTVLSYKDKLCDITVFPRNTGRLGGPVNRADVTPLKYQPVHEVETPPRVS 1366 DDWFQFYNGSLVIDLEGKKEVDIEMSISAGPNFEMYEQTVAPKCGNVTTSFTFLSYQKHLQDITRFPMNVGRLGGPVNKAQVTPNPFTPKEPVASP---S 1336 YEWLTMYNGAVTFDLRSGADMELFVSIAGGDDFEMFEQTVPPKCGSVSDSYTVLSYADDVKSVTEVPNKTTYL - - - - - -ADEQPTTSAPRTSIVNT- - - - 1296 KSWILNYNGTLNFDYRGVDDFNVTVDISAGDNFEFSVRTVAPKAGKVNESFTKLSYSNELVDIKKPLTAAGRLKGPFN- $-\ldots$

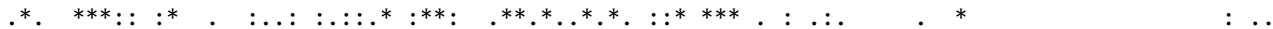

DRRRDAEGRELGPLNKSPVEGDRAFTKE -GDPIIFRNGQWD-- - - - -YEEDDVTSQMNC- - - FPRGLKGLKESADLFETRDTCSKLADVIDFSHETV 1453 GKRKMA - - - - - GPKDGDRAFDQDSGKPIIFVDGNWE - - - - - FEE-EVTAQAGC - - - F- -GLGAVGETVKEFKERGTCGKIADIVDKGHGSL 1411 - - EDD - - - - - - PPTEGEIARTTN-GTLVQYRGGAWKPMVERTPTMSKKQVGPELTVSDPQMYKCIKNMNKNVKILTDRQCTAKLANIVDSAQELV 1382 - - VPKETPKESSDDKDKSNQKRKGAMDSLLNAVAQMETINSDAN-GC---F--SLGGLKSTAKMLDSRKTCEKFADIMDFTHDTL 1431

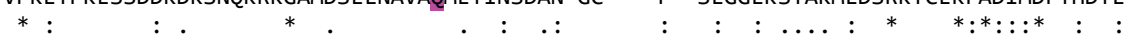

TEKGFSQD-1-1 GSNSTFVEDLAVGAKQIRKFGESLDVFEGSMSAAKTAELIDNTHAAFSGPADGSPISNVVQLLLPMLSSIKGMSGKMESKMASLTAMFQPCKKAITHLIE 1482 GVKD-1486

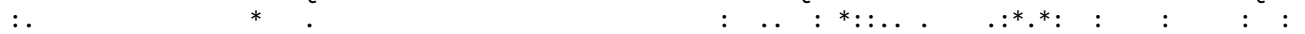

ESVPGTVLSMVENQQYTWATLLTIVGGASLLWTSKSEMKFYKKLSVFAMIVWSPFLASKTWTLGKWVKTKFTNFFHRENIDEETCRKHSMAGAFEEVKKY 1607 ESIPGLITSAIKNENYVWATLLTLLGGTSLLWFCKSKKSYIKKLSIFCMIVWGPFLAHSVWKFGSWIKKNAFGMMKKK-FMNETCQKHSMAGMFEGVKET 1564 RSFPYLACKGFKTDKWTWAALASILVGAALLHYYRSDLKFVKKWSVMCMI IWAPLLAEKAYHLGTWIKEKFLKSLPRTRTIKDSCRKHSLAGAFECLASA 1582 ETIPGLAIVDFKKGKYMWATLLTLIAGAALFWACKSOKSFLKRFSVVVMIIWSPFLAGKVWSLGOWIVOKWCHLWPKS - - -DSCROHSLAGLFESAKTK 1582

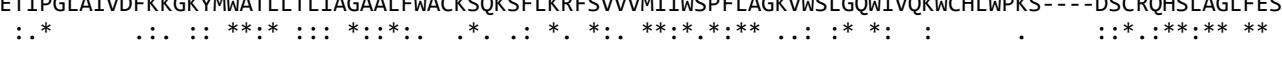

LGNFSEWFSDNWMSTIOALLSLLCTVASLIVWGTMPDTKOLNSFTEKFKVAGEKGRSFSNIFSGFNSITKMSNEMSSAFMKWTMOGMKDSLPNADSALOT 1707 FGNFTDWFSSNWIVAIOGLLSLLGVVASLIIWGSIPDDKELNSFSAKFKAAGDKGRSFTNIFSGFSNINKTSSEWSOKFVSWIMSISGTSLPKADSALOK 1664 SCAY--- IKDNWAKTMSSLLTILSVVASLVMWGKIPDDKEITSFADKFHSIGKKGRSITNIIGGFEKITSVCKKWSETLVSWIVSNVSGGIPKEDLAMTA 1679 VRGFPDWFRSGGMNIVTQVCSVLLTIVSLITLGTIPSAKKSKSLADRFIEFGNMNRAATSIAAGYKSISELCSKFTHFVATHFL---GATVDDNVFKD 1677

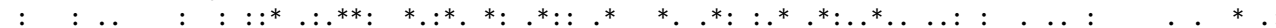

LVTFDIREWIKETRVMALQENRFQGFGGEEHLVRVRHLYDKSQAIEQALASGVKV-DTQLSMIIKDCKEKCTELKNESYTFKGMKKPRIDPLHVCMLGKP 1806 ILDFNIKEWVEEVRQMALQENRFKGFGADEHLIKVRRLYDRSMKIQTAIMDGCKI-DIQLGHIIKECKDKCNELMNESYSFKGMKKPRIDPLHVCMIGKP 1763 YLGFKIHDWVRETRDMALMENRFRGFGGDEHLVKVRRLYGHSLKIDNALMEKQIVPDMQLSLIIKECRQKCLELMNESYTYKGMKQSRIDPLHVCMLGAP 1779 LVTFNVKDWVEOVKVASLEENKFKSFGSPEOLTRVRHMYDKSLEITNKLLDRNKVPVAMLPVIRDTCKK-CEELLNDSYSYKGMKTPRIDPFYICLTGPP 1776

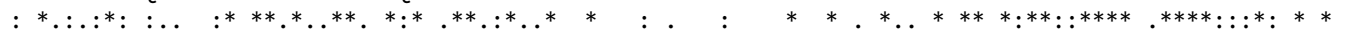

GVGKSTITHVVINNLLDHRGEPETDRIYTRCCADAYWSNYHQEPVVLYDDLGAIKSSLKLSDYAEIMGVKTNDPFSVPMAGVEDKGKHCTSRYVFSCTNV 1906 GVGKSTITHVMINNLLDHRGEPDVDRIYTRCCADSYWSNYHQEPVVLYDDLGAVKSNLKLSDYAEIMGIKTNDPFSVPMAGVEDKGKHCTSRYVFSCTNV 1863 GVGKSTIAHVVINHLLDHRGEPEVDRIYTRSCADAYWSNYHQEPVILYDDLGAIKSNLRLSDYAEIMGIKTNDPFSVPMAAVEDKGKHCTSKYVFSCTNV 1879 GVGKSTVASIIINDLLDYMGEPKTDRIYTRCCADSYWSNYHHEPVIIYDDLGAISKVASLSDYAEIMGIKSNRPYSLPMAAVEEKGRHCLSKYLVACTNL 1876

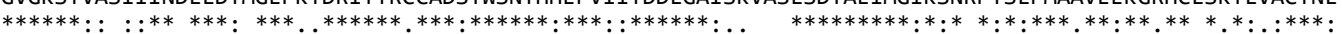


BnRV1 LELDDSGDVVSKLAYYRRRNILVEVERDERIPRDESNPTAGLVFTVLGYHIVDER-- - - - - SGRVAFGIKETWEEPFLRSIDTSQWRFERVPYPVFL 1997 BVCV LELDDTGDVVTKLAYYRRRNVLVEVDRNFDIPRDESDPTKGLVFTVLGYNVTGVN- - - - - NDRVNFGVKDVWDESFLRDVNTDNWRFERVSYKTFL 1954 RTSV LNLDDTGDVVTKMAYYRRRNVLVKVERDPDVPKNEANPTEGLVFTVLGHDQNC-1- - _ MCDV THLDDTGDVKTKEAYYRRINLPVTVERDLAMPMSPEDPASGLLFTIGDIHENGRNVSVVESRLLNGRVPFRAGD-1........

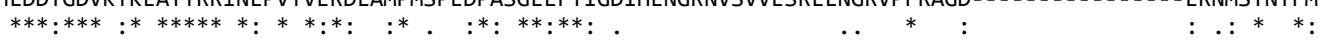

BnRV1 RFLCVYTDAYMESOENLLTGIT--CYRPDMDEIPTLDEIFP-_-VVAQAGGA-

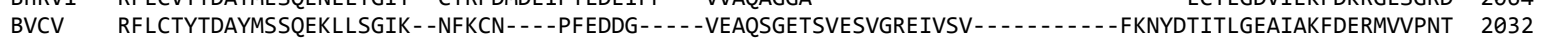
RTSV RFLCMYTDAYMYSQEQVLQGIK--TFKMN----PFAPEPEF---AQAQNGEAAECEIVEEMQEVPGEAPQEAKELVKIETAPNMDELVEAFNKLRVTPGH 2059 MCDV EFVRIYATIYMENQQQLVAKLSGDDYESSSSSFPENEELEFDFLAQAHNGV

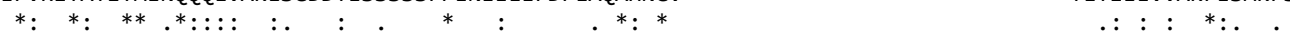

BnRV1 LCASFQKRGSKYFPPDAWGTS--KSMSFNKLILTLCECSDQGVCNFDIMMSQIRELSVQKDVKRIVKAIRVHKLKADPLETEMMVNSNGVEGL--FEDVD 2160 BVCV FASFF--KGTRFMAPEKWQSR--KVASFSTLLANLCGCSTGNHCNFDLYFKHLIEVCKAKGVH---QNFVLGQLSLVPEETTLVIKKA---DF--FSDLR 2120 RTSV LNDIL--RDGSGCYIDEWAIAGPRWLSFHELLPFTCGCHHTRVCDFNIVYNNMCKAVRSOSVH---FKYRANOAIKYAYTHKLHSOCRYSIDFEKLRECN 2154 MCDV LNAEI--EKFERIGVDGWRTN--KALSFNDLVKRFCGCCLGDDCNFDFHYRTLFKVLIENKQIPAYKCMVLHKVNPDRMKTQIKMVNGYTLET-MFKTLN 2126

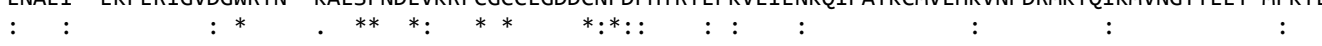

BnRV1 ALTFFSMLACWYRWSL-PGYMCV-YRINQQKSAQRFAEKCRDEVENEDRIPNCVEIKVEDGNSFVWDKAMKFFPSCLSAIGCVPLFDGCEYYLASTSLSM 2258 BVCV DIDIFVALASYYRFSF-RSDLCF-FHFISKPSNKAVSHVGALDLDFDERLP-LESLDHPNGDAFVWPSVRLVFPTLIDTIGCVPLCVNGKFVFLVEKVDS 2217 RTSV PLDVFVCVLSKYT----ADDHSFERRCPKKMNVVRMQRPPVFELKMRPPSDSVVVEDEQGQRIFEWPHLYIFL-.---RYRAIEFKDDKGSLTVREDAGA 2245 MCDV PLTIFLYLVFVLKCGISADNVCLSYQLFAMNDAEQVE----FEIEDSLRLD--EQVQIGQYSCYVWPSVGKFYPEILAKRGCIAVNDGTTFYIFVSSSQI 2220

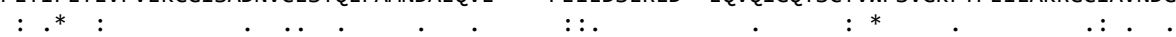

BnRV1 QRL--EERRSKIWELIWANGVEISCDPLSLL-PRRERLTIAGIVNGISSFGTAEEPSGEVKQVYQSIKENYGLGNIYLTTIILVAQAHSDRVKEMEMTKN 2355 BVCV KFV--PVPSCAKWKDVWSKGFEQNVNPLSLL-PQAERDIINCSLKNICAFGSFDEPSQEVKEAIDQISRLYGEGGVLYTFVLLLAQENARRTAFFSRENE 2314 RTSV DVC----PWNEFLKLPWLDGDQL----KSVL-PAHLHRMVQARLEOVEIMEENGNYSGEMRNAIAEIKEYLDQDHQWVAALVLVACA----VKERRRMTH 2332 MCDV DKIHPEAAWSDMLQGVGRRGVDI--.-LSIAGPTKTKFLIKHVESCYETLKSPEDWKAKCKEYYESIS--...-LYEYILLLMAVGSRAGIETQRMSK 2308

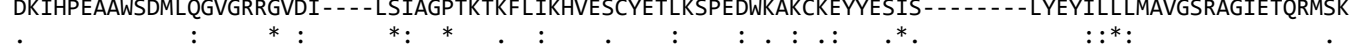
2308

BnRV1 NAKKEKVFSAYEKLORY ---EKSVVEGISGKMKVFLSIAGGIAATGTIVGLFFLVKK - -LFSPKSEEVGAEEIEVEDAEAEMSGAHESGMFKTAHIKTRS 2450 BVCV FQTKRNAFRASETFKEY---EKAIAGNLSTNAKIALAIGAGITASGILIGLVFGLKS--LFNL----IKGDDDEEDNAEKEASGAHESDMFQTNFVKTQR 2405 RTSV DKLHRKSFNALDKLDAW---YTTTAPKTSKKMKILLAIGASVAVAGVAVGAVILLQKTNLFGS----KEDEEIEGEEGETQASGAHESDGIVTQHLK-RD 2424 MCDV YQARKNKIRMPEVLEKYIEVEKATIGKLSKPAKTCLAIGAGVAIFGVLAGLGVGLYKL-ITHFSKTDSEDNDIEIDDLVPEMSGAHASDENVTTYAVRRQ 2407

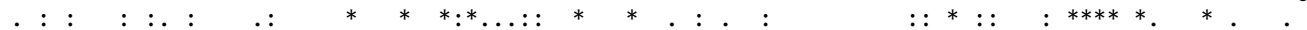

BnRV1 F--PVRVSR-- - - AKAEMMGAHESGA--FRTEHVRRIQRPYVQKAQAHVRGGVHYDEVSELKRDLVIEKRKKKRNLAIKAAS MKPQIRIVN--

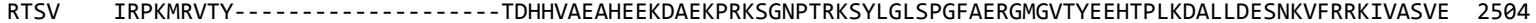
MCDV V-PKVRLAKQFKVRSSPSPSDNEQPKVDILVPEMTGCHASDE- - - HLTKHFTKRRVTMKRVGAVKESHIVTYDENTPHVRLI----RNLRRTRLARAIK 2498

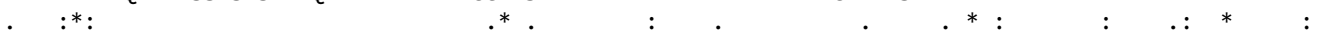

BnRV1 QAVKKDKRSVRTRDSKDA-IDLWONNSLIQKGMLASNG-EMEDVEKSLIERKIL-REEKHSAEGSYITGDPEFRVHYEIVEELEKTRKAVLRRSKELIENG 2621 BVCV HKIGNSKKKV--PDVVVG-IDKWODKVKOLGILPGES-ESKGPLSSIAKGNMV-AGESOAAAGELLVGDPDFLRNSEVQDMLSKMICIDTDEFSALIODG 2576 RTSV SAVKQGGKAS--KDTVLSQIGDWQDKVKATGVIAARQLEASGSLKKIHNLNSR-RTSSHVMPG-LVVHDGAFERSDEVDAELHRITIDEVKSCPKMIKEG 2600 MCDV QMAQLGEL----PDTLSE-IQVWQQYVVDKGIRPAEHTTDFRLFSAIADQEQEDPEEINMASGETMKFDEN--KYNEIVQVVKGISPTKSD-IVTMTTKG 2590 $: \quad * \quad * \quad * \quad * \quad:$

MHVTVEKQSTIGSF- - - - - - - - - - BVCV TQNVVTKQAHVGDY-_......-GLTRDVNMVELLQTHVSKMSCTILRVNEDKCVSYGVLRLKGTVVLAPAHYFEEFSEEDKLYFVCPNKVV 2659 RTSV VSTLSVKKASVGMLALOKAESOLSFPFTSRAGVDRDLSMTNLIDTHMAGMSCIIISELGNVFRTFGVLRLCGTYVCMPAHYLDEITSEHTLYFVCPSKIT 2700 MCDV AHHTAIKQVRIGYK---.-.-.-SLDKDPNMVSILSNQLTKISCVILNVTPGRTAYLNVMRLCGTFVVCPAHYLEALEEDDTIYFISFSVCI 2673

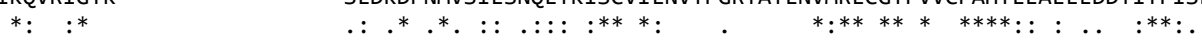

BnRV1 RIQLEMSRVALVSAHQDLVVWLGKSAPPSIDYMSHIPTRKDWEAYRPCSGALAFTEYTODMTLOMVSALDTIEMTTTNVEVPTAEYEMLESTHTVILGL QIPFVANVSLVSDIO RTSV QIQLERHRVCLVNGFQETVVWDLGPSVPPSRNYIDFIANADDWKNYKATSGALVMSKYSVDSMLQCVHFLDSIELTEANVSVPTSYYEANGGIHTIISGL MCDV KLRFQPDRVTLVNTHQDLVVWDLGNSVPPAIDVLSMIPTVADWDKFQDGPGAFGVTKYNARYPTNYINTLDMIERIRADTQNPTGIYKMLNSDHTITTGL

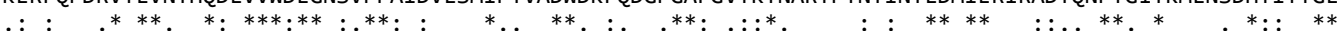

BnRV1 RYRVHCMVGFCGAAVVRADAKAIRKIIGMHVAGNVSRGVGYAEMLIKETIQTAIDALSKE - - - VVQKAMDEPAMKV- - - CEKQCATIEGKGNIGQIGM BVCV RYRVHCMPGFCGSAIVRADTRAIRKVIGMHVAGHKOKGVGYAETLSLEPILEAIKRVCPT - - - -VVERSPVNNGIEK-- - CEKQCVVLEGKGNLGILGR RTSV RYRVHCMPGFCGRAIMRADATCFRKIIGMHVSGLRNKCMGYAETLTQEHLMOAIETLKETGLLKHIPKGAIGAGEEKLPEHSKKQSLSLEGKGNLGIVGQ MCDV RYQMYSLEGFCGGLILRACTRMVRKIVGLHVAASANHAMGYAECLVQEDLKHAINKLSPD - -ARSLIIGHLNPKVET-- - ATKQCGIVRSLGSLGCHGK

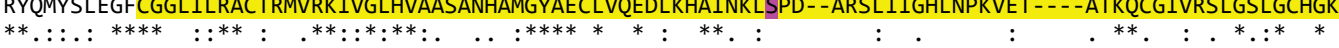

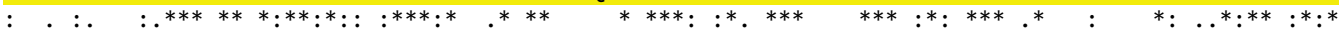
INGIDGSDFWSPIEMKTSPGYPYILKRPSGAOGKKYLFEELEPYPSGRPKYAMKDPELIENYERIKEEVTSGVKPSIMTMECLKDERRKLAKIYEKPATR 3099

MCDV INGIDQSDYWLQIETNTSPGWPYTKRKPKGAEGKKWLFKEVGNYPSGKPILEMEDSGLIESYNKMLRDAKQGVAPIVVTVECPKDERRKLSKIYEQPATR 3067 
BnRV1 SSIVHRDGIAGAHLLRYSQGMPSGFSMTVIFNSFVNYYYMALAWMYIVSRSELSPQADLGSFDRFTKIIVYGDDNVVAVNDAFLDVYNLQSVACYLSLFG 3295 BVCV SSIVHRDGLAGDVLLRYSQGMPSGFSMTVIFNSFVNYYYMALSWINIIANSPLSPQADLVSFDYYTKIVVYGDDNVVAVGDEFLDFYNLRTVASYLSSYG 3250 RTSV SSIVHRDGICGDLILRYSQGMPSGFAMTVIFNSFVNYYFMALAWMSTVGSSLLSPQGSCKDFDTYCKIVAYGDDNVVSVHEEFLDVYNLQTVAAYLSHFG 3299 MCDV SSIIHREGIVKEYLFQYCQGMPSGFAMTVIFNSFVNYYYLAMAWMNLISHSPLSPQSTVRDFDNYCKVVVYGDDNIVSVDLNFLEYYNLRTVAAYLSQFG 3267

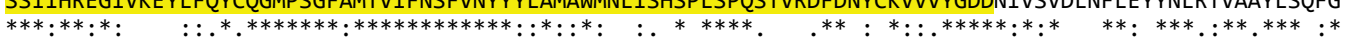

BnRV1 ITYTDDAKNPIHLSEPHVPIDSVTFLKRSFVKL-DKSGSLWKAPLDKASIEERCNWIRECEEPEEALNONLESALYEASVHGEGYFNDLKTRVDGALERV 3394 BVCV ITYTDDAKNPIHLSEPHVDITSVTFLKRAFKRV-DNTGCLWKAPLDRTSIEERCNWIRECEVPQEALYQNIESALFEASIHGKDYFMDLKNRLDFALDRV 3349 RTSV VTYTDGDKNPIHMSKPYEDITKMSFLKRGFERV-ESSGFLWKAPLDKTSIEERLNWIRDCPTPVEALEQNIESALHEAAIHGRDYFDDLVRRLNSALTRV 3398 MCDV VTYTDDAKNPIEKSVPFVEITSVSFLKRRWVPLGGRLSTIYKAPLDKTSIEERLHWIRECDNDIEALNQNIESALYEASIHGKIYFGDLLQRIRIACDAV 3367

BnRV1 MLPTRSESFKSCQVRWWSNMTGAMLSQPSLASLVELSNKNHIDLGFKFKNMALDGAETTLGQALGMAKNSPFIYYDV BVCV MLPKTKESFKQCQARWWSDMTGAILSQPALTSLVELSKKNQIDLNLQVKDHLV-GTSMSLGDALSRAKHSPLVLFEV RTSV MLPPTDISFEECOARWWASVTGA-LRAADYTSLVRRASSGHVEFNKKYRDMFR-QQDLPLKEILMKSKPVALLDLEV MCDV MIPVPSVTFKDCHKRWWASMTGGALDPASLSRLYLAAENQLVDTRKVWKDRFL-GEDRSLIDMLKSARAVPLAAYHV

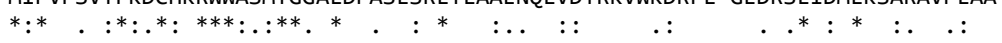

BnRV1

BVCV

RTSV

MCDV

CNDV

PYFV

CaTV1

MYMoV

LNLCV

ToMarV

ToTV

SCLSV

ALSV

CRLV

CuLV

AVB

SLRSV

SDV

CLVA

DMaV

BRNV

SMoV

BnRV1

BVCV

RTSV

MCDV

CNDV

PYFV

CaTV1

MYMoV

LNLCV

ToMarV

TOTV

SCLSV

ALSV

CRLV

CuLV

AVB

SLRSV

SDV

CLVA

DMaV

BRNV

SMoV

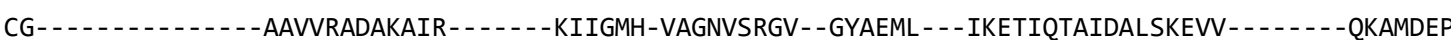

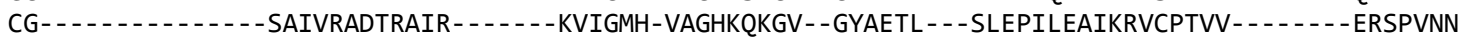
CG- - - - - - - - - - RIIGIMRAD-VSGLRNKCM- -GYAETL - - TQEHLMQAIETLKETGLLKHIPKGAIGAGEEK

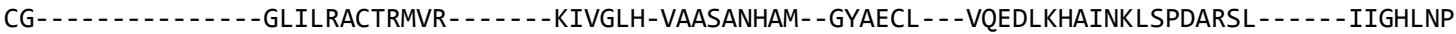

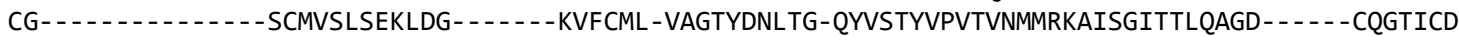
CG - . CGCG CGCGT - - - - - - - LIVGIH-CASYDGVAAERGFISSN---ATAIYREQLEDL - PTGPVK - - - - AAMVRCD

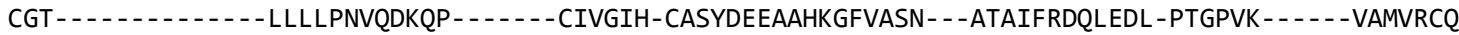
CGS- - - - - - - - AVLIPGAINGQP- - - - EIVGIH-CAGFSDAMQRKGYRGTT-- -AALIFYEDIEKYLPSVDLQ- - - - EAQTNIP CGSLGSKPMPACYSYTFDTFAGLCTSPLISMDGGRCVLLGLH-VAGDKSKM- - -GY- - - - - - AQIVTLEDFSDINFSEK - - - - - - - VGQGP - CGSLGSKQMPACYSYVFETYAGLCTSPLIAQEGGRCIILGLH-VVGDRSKM- - -GY- - - - - - AQIVTLDDFSDVALSDK - - - - - - -VGQGP - . CGSLGSLRMPPCYSYTFDTYPGLCTSPLICMSGGRCILLGLH-VVGNMSKT - - - GF - - - - - - SQIVTLDDFSEVPLQSGTL - - - - - VGEGP - -

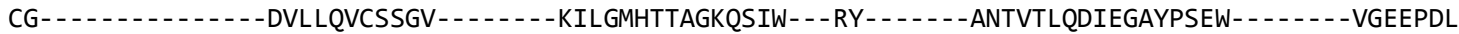
CG - . - - - - TPLVANYGKGKGLKIASIHVVHHFSATEKDTIIAGSGSLLTKEEYLEASLLLGDIKHPLETDRIQ- - - - - ASGCLSG CG - - - CG- - CG - - -

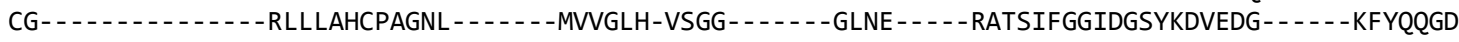

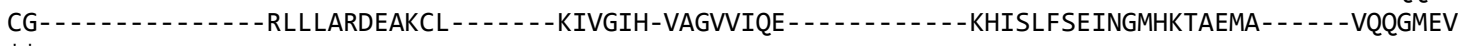
$* *$

AM- - - - - - -KVCEKQCAT - - - - - - - - - - IEGKGNIGQIGMIEDKLLPKMPSRTTICKSLIHGLI - - - - - G-DVVSEPSILSKWDRRLGEK GI- - - - - EKCEKQCVV- - - - - - LEGKGNLGILGRVVQSAVPNVPTKTTIAKSLIHGLI - - - - -G-EVRTEPSILSKWDKRLGDK LP - - - - - EHSKKQSLS - - - . - - - - LEGKGNLGIVGQLTAQLVPTSVTKTTICKSMIHGLI - - - - - G-EIKTEPSVLSAWDRRLPFP KV - - . - - - ETATKQCGI - - - - - - - - - VRSLGSLGCHGKVTSEDVAMTATKTTIRKSRIYGLV- - - - - G-DIKTEPSILHAHDPRLPED SPVSEVVFSTLKMDELLSSTPTPSGNLGVFKPNDRMGLIEVVGRIFPNTTPKAICRSTIIPSLIQRFM- - PR - - - KPLTEPAILSPLDGRLGEC SPISDTVAETIKVDQLFSSKPGASGKFGVFGVNDTIGIIDVVGRTFPETTPKSITKSTIVPSLIQPYM- - PR - - - KPLTEPAILDPRDVRLGEN LL--- - - -KQLRVELEN- - - - - - - -PFQIKQVALLGQVPNELSVSVPHKTTLRQSELFHVL - -SEEIG -PHLTEPSLLIKGDRRCTKE QL - - - RQLRQVDTN- - - - - - PFEIKQVALVGRMPPELAVNVPHKTTLRKSEIFEEL - ETHLG-PHLTEPSILTIRDTRAASVL--- - - KKLRASTTN- - - - - - PFEIKQVAFLGTVPQELAINVPHKTTLRKSEIFDAM- - TKVLG-PHSTEPSILTHHDDRPEVE IL-- - - -KSIRSRETQ- - - - - - LFEENQVYYLGTVPQELAATVPHKTTLRKSQLFEAF- - - - G-PAETAPSILTVHDKRGDG-

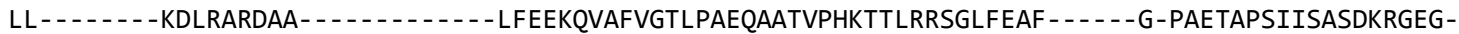
AI - - . - EEFFQS - . - - - - PFDTKQVFCLGKVPRELAADIPHDTALKLSIAHDIL - - TEVVG-PCTTEPSILTSRDKRLSGK -

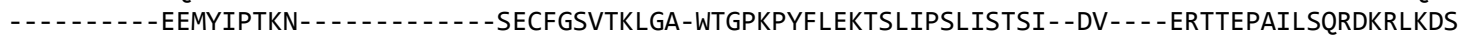
- - - - ECYCIPTAS- - - - - EMTTTEPAILTTKDPRLAHS PI- - - - - SNTSR - - - DE- - - IL-- - - - -DRFSIPISECR - - - - - - KELTPMTTRLGY-VVGQYPRALRKTSIVPSIIHDNL - - - -WR -KPETEPTILGKIDDRSPFP D- - - - AEFIYFDAE- - - - - - EEITPMVSKIGYV--STAVPQLSKSQIEESPIFDSL - -CQKLG-DPKVAPTILSKHDPRPPTP TPLLD- - - - - VHMKELIEA- - - - - - - ERVTDMVDKIGRVDSSQQFRPSQGTSIIKSEIHDDL - - -WR-RAETCPTVLTRSDPRPEVP

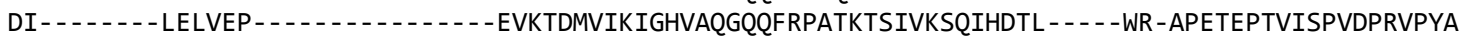


BnRV1 R-GTWDPVEDAVKKYGIMVVPFPR--EEVKEVEEHLTRVFAKRHNSLNK- - - - - - REVNTLEIGINGIDQTPFHSPIEMKTSAGYPYVLRTPS BVCV R-GEWDPVMEAVKKYGVATVPFPP--NEIQEVETHLCGVFQNFENSLRK-- - - - REVNDVEVGINGIDRSDFWSPIEMKTSAGYPYVLKKPP RTSV P-GEWDPMKDAVKKYGSYILPFPT--EEIQEVENFLIKKFRRKENSRRT-- - - RNVNSLEVGINGIDGSDFWSPIEMKTSPGYPYILKRPS MCDV CNDV PYFV QIGKWDPVFEAALKYGTRIEPFPI - -EEILEVEDHLSIILKGMDNTLKK- - - - - RNVNNLEVGINGIDQSDYWLQIETNTSPGWPYTKRKPK R- - YDPMIEGIKKYQDQAKPIRG - -NWRKRIIDSMREQMEDWETFMVRENYD- - - TLDLPMHTIINGISGIEYYEPLNMSTSEGYPLILSRPS R - - YDPMIDGIKKYEEQARPIKI - -SWRNQIIESMAAQMQDWETFMVREGYM- - - TMDLPMSVVINGIDGVEYYEPLNMSTSEGYPLILNRPK G- - -FDPYVAGVQKFNETACCFDM- - RVAETVMESMSDDLLSKLANIQVPGGK - - - PVVRSEEQILNGIPGEKYYDSMDFSTSCGYPFMIM-GF - - - -FDPYVKGVEKFNETACNFDN - -EVAQIVMQHMKASLLANLERISVPGGK - - - PIVRGEHEILNGIPGEKFYDAMDMSTSCGYPFSLS-EF $\begin{array}{ll}\text { LNLCV } & \text { G---FDPYVKGVEKFNETACCFDH--ETVEVALQHMSEDLLVELVKVDVPGGK--- } \\ \text { ToMarV } & --- \text { FDPYVIVRSELEILNGIPGEQFYDSMDMSTACGYPYTLS-EF }\end{array}$ ToMarV - - -FDPYVAGVMKYNETACGFDD--DIAKLAFENLKCSLLPIMRSQKIPGGR - - -PCERDEDVVLNGIDGCDYYDGMELSTSCGYPFNKM-GM
TOTV - - -FDPYVAGIQKYNETAQNFDE - DIARLAYEGLRQAILPVLHSQRVPFGK- - - PVTQNEDVVLNGVDGFDYFDGMELSTSCGYPYNKL -GM A- - - FDPYLAGIAKFNETAHSFNM- -HVAQEAFEYMKRRLLRHLSKIPVPGEK - - - PEVRSEMVALNGIPGEEYYDPMDLSTSSGWPFNKG-ER NNKDFDPFLSGMGKYAVEAHSFD- - -EDEELFEDALDRVFSEIPMFT - - - - - CEDLSNDQVCNGIEDDDYAEGLVMQTAEGYPFCTMRPP INPEFDVFLEGMKKYAVEAHSLD- - -EDLEVFEDALDRVFLEIPEHA- - - - - - CEDLTNDQVCNGIEDDPYAEGIVMQTAEGFPFCTQRPA NDPDFNPFKAGMLKYAVEAHGFN- - -EDEEFFEDALDRVFSEIPDFN- - - - - - CQELADDEVCNGIEDDPYAEPLVMQTSEGYPFCTQRPA GHEGFDPFKDGMLKYTRAAGPFDEADEDFQDALDDVFLDIGDLRTEKEIEQNQSKAICRILNENDTLNGGIEQDFEDPIVSKTAEGYPFCCQRPP N-- - LDIFKKGMMKYKAVAADMSPVTDEEEKIWNVTWDSIFDLPGGIQSK-- - - - CHLLSEDENLNGVNGDNEYRGMVVSTSEGWPEVLNRKN - - - -YDPYATIGEKFVQEVGPIDL - -SVGSDASLVVANIGSSWKAVGKPQC - - - - PTVLTWEVAINGDAAIPYCERLPLSTSEGYPDSIQRNF - - - YDPYSAGIRKFDKEVGPFDW- -SEESDLQFACAEILDTWQTKKPEKFAI - - - ETVCSLDVAINGVHGLPFAENFPIGTSEGFPWVLDRAR - - - - FDPYLQGIHKFEKQAGPFDF - -SEESDLDLAQKNISSEWERFRPEQFDV - - - DTVTTLEVAIQGIDGLDYAESLPIATSEGFPYILERRA - - - - FDPYQAGIRKFEKEVGPLDF - - IDEFSTESTVLVDIAEELNNKKREVGGF - ELDTVLDNHAAINGVEGVEYAEPLVMGTSEGYPYVLERQP - - - -FDPYTAGIMKFEKEVGPLDF - -SDPDTPESTVIEDISEELLREKKSLGGF - ALDTVCSNEVAINGVDSVPYAERLVMSTSEGYPFVLSRKA $*$. *: * *

BnRV1

BVCV

RTSV

MCDV

CNDV

PYFV

CaTV1

MYMoV

LNLCV

ToMarV

ToTV

SCLSV

ALSV

CRLV

CuLV

AVB

SLRSV

SDV

CLVA

DMaV

BRNV

SMoV

GASGKKWLF - - KEVGQYPSGRARYEMEDPGLIASYDEMLGQIKRGVAP - TFITVEHPKDERRKLKKIYEVPATRTFTILPPEVNILFRQYFGDFA GATGKKWLF - -NSVGCYESGREKFEMKEEQFKTSFEAMRTQILNGVVP-NIMTMECPKDERRKLGKIYDNPATRTFTILPPEINILFRMFFGDFA GAQGKKYLF - -EELEPYPSGRPKYAMKDPELIENYERIKEEVTSGVKP-SIMTMECLKDERRKLAKIYEKPATRTFTILSPEVNILFRQYFGDFA GAEGKKWLF - -KEVGNYPSGKPILEMEDSGLIESYNKMLRDAKQGVAP - IVVTVECPKDERRKLSKIYEQPATRTFTILPPEINILFRQYFGDFA DAHGKEYLFEICEDGSRAIK - - - - - SAKLNANYEAYGSSLSSGEPF-PLISIECPKDERRALDKIYDKPKTRLFSILPVEFNMHARRLFLDFN DAHGKEYLFETMESGERRIK - - - - - SAKLEAHYESYGHALOSTEPF - PLICIECPKDERRALDKIYEKPKTRLFSILPVEFNMHARRLFLDFN GKNKREFLD--GEPGYYLLAR - - - - DKPVYEEYMAMDDAISQGIVM-EMVTCECAKDERLPLEKIYEKPKTRLFTILPFHYNMLVRKYFLDFS GKNKRGYLD - -GEPGDYMLHR - - - - -SRPVYEEFCRMDDEVRAGIVT -EMITCECAKDERLPLEKIYEKPKTRLFTILPFHYNMLVRKYFLDFS GKSKRGYLD--GTPGEYVLHR--- - - ERPVFADFIELDESIRQGRIT-EIVSCECAKDERLPLEKIYQRPKTRLFTILPFHYNMLVRKYFLDFS GMNKREFVQSTGEGERVELKR---- - -DTPVFEAWEELDVQIRKGIHV-DLVTTQCAKDERLPLEKIYGKRKTRLFEILPFHYNMLVRKYFLDFS GTSKREFVEPSGDGDRVQLKR - - - - TTPIFDDWEALDVEIRKGNFV-ELVTTQCAKDERLPLEKVFGKRKTRLFEILPFHYNMLVRKYFLDFS GKSKRGHVV - - EVEGVHLLDR - - - - - TSEAYTAYIELLQSLADGEVP-VMVTSECAKDERLPKEKIYEKPKTRLFTILPFHYNMLVRQYFLDFS GVTGKTWLF -AGSPGDWHIVP- - - - - NSLLANELNVKELNLSKNIFE - PVIGIDFPKDEKVDSSKVYIKQKTRLFTILPVDYNILVRKYFLSFV GASGKSWLF -AGAPGDWHIVP- - - - - GSLLANEMHKKEVAPSRGLFE -PLIGIDFPKDEKVDSSKVYIKPKTRLFTILPVDYNILVRKYFLSSV GATGKSWLF-CGSPGDWHILP- - - - - GSLLHNEMNKMERNLSQGIFE-PVIGIDFPKDEKVDKSKVYIKPKTRLFTILPVHYNILVRKYFLSFV GKSGKGWLL -GGIPGDWSLKK - - - - - EGPLNDAIEHLEDNLANEVFE - PLIGIDFPKDEKVMRAKVEVKPKVRLFTILPFHYNLVVRRFYLDFV GEAGKERFL-LGLPGCYTLNR--- - - NLPMYQRILDLD-ALSATTIP-CIVGLDTAKDERLPLSKIYQDVKTRLFTILPMEYNYLVRKYFGSFV GEKGKKRFF - -DLKGENVRVP - - - - - TPALMEELEVLERELOKEEVCLTCINTACAKDEKTAPKKVRVOPKTRIFEILPFOINIIIRRYLMFWM NESGKERFFEEVAPGHRVP- - - - - - RGSWVEDIAEIEEAAYRGDY - - LTTTITCAKDEKTKLDKVFVNPKTRIFEMLNFCLNLVIRKYFFFWM GDTGKERYF -EEINGKRIP- - - - - - KGDWVQDIDQIERAAVAGNL - -EIYTMACAKDEKTLKKKIYETPKTRIFEILPFTFNLVIRKYFLFWM GDVGKFRYF -SKNLYHWEL - - - - - - NEGPAKELDELEQSVAREDFDGKIITIACAKDEKTKLIKVYEKPKTRIFEILPYHYNILVRKYYLFFM QDTGKFRFF-DKDGDRWVA-- - - - - KDEVLDDLKELEEAIKSDDFKGGIITIACAKDEKTKMKRVREVPKTRIFEILPFHYNILVRKYYLFFM

$$
\text { : }
$$


BnRV1 AMVMENRFDTFSQVGINPESLEWSELMNSLMKKGKRGFAGDYAKFDGIGSPEIYHSIVSVVNAWYDD- - - - - - - GEENALARHALISSIVH BVCV AMVMTTRFDHFSQVGINAESMEWSEMMNGLLAKGKRGFAGDYAKFDGVGSAEIYHSIVNIVNSWYGD - . - . RTSV AMVMSTRREHFSQVGINPESMEWSDLINSLLRVNTKGFAGDYSKFDGIGSPAIYHSIVNVVNAWYND - . - . - - GEVNARARHSLISSIVH MCDV AMIMTNRSKLFCQVGINPENMEWSDLMHEFLHKSTHGFAGDYSKFDGIGDPQIYHSITQVVNNWYDD - . CNDV VFVMANRHKRGIMVGINPHSREWSDLAISLASFSPYGFNGDFANFDGMFHPTSFDMVAELANIFY PYFV VFVMANRHKHGIMVGINPHSREWSDLAISLASFSPYGFNGDFANFDGMFHPSSFSMVSELANIFY - . . . . CaTV1 ATLMRAHNEIPCKVGINPEGIEWTTLANGFVEKSSVGFSADYSSFDGRAPVFIFQWFCDMVDKYYGDKP - - - - - GSENSLARHALLMMASN MYMOV ASLMRAHGTIPCKVGINPEGFEWTALANSFLEVSQTGFSADYSSFDGRAPVFIFQWFCDMVDEYYGDHS - - - - - DSPASLARHALLQMASC LNLCV ASLMRAHNTLPCKVGINPSGLEWTVLAKNFSETADVGFSADYSSFDGRAPVFIFQRFCDIVDKYYGDLP - . - . - GSENSLARHALLMMASN ToMarV ATLMALHNAIPCKVGIDPTSSEWTLLANGFRAVSDVGFSADYSSFDGRAPVFAFQWFCDLVDEYYGSKP - - - - - GSPDSNARHALLMMASC TOTV ASLMASHNALPCKVGINPGGIEWTLLANGFRAVSDTGFSADYSSFDGRAPIFAFQWFCDLVDDYYGSPP - - - - - GSPDSNARHVLLMMASC SCLSV ASLMRSHNDLPCKVGIAFDGIEWTTLANQFLAVSDQGFSADYSSFDGRAPIFVFQWFCDLVSEYYGDDL---.----QGESARIRRGLLMMASS ALSV SQLMALHNEVPTKVGIDPISNEWSILCHSLHSKGTNWFNGDYSRFDGITPRNVLQGIVKRISRRYANKSSFAITDPTLSINGDLARSLLMDMCST CRLV SHIMTQHNTIPVKVGIDCLSNEWSILYHQLRSKGTNWFNGDYSRFDGITPRNVLQGIVKRINKFYNNKNSLAITDSNLSINSDLARSLLTDMAST CULV SQLMALHNETPTKVGINPLSNEWGILQFALSAKGENWFNGDYSRFDGITPRSVLLGIVKRISNRFVNKNSLAITDKTLSINGDLARSLLMDMAST AVB ARLMQKHNETPVKVGLNTSSLEWQHLYDQLSAVGFNWFNGDYSRFDGITPRCVLQGLAIRISKFYRQDEAQGLVSKK -KIRTSVAHSLLLDMANS SLRSV AELMKLHNCIPTKVGINPLGYDWTILGKKMHAKGTNWFNGDYSRFDGVTPRCLLIEISNRITKLY- SDV QLLMVAHDELPSKVGINVYSESWDRLLGRHTRLANH-FTGDYSGFDTSTPRVLVYAIIDKINELADD- - - - - - GEVNQRTRRNIIRFVLN CLVA QWMMSLHSDLPCKVGINPFSYDWDIMAMKHSAYANH-FCGDYSGFDTNTNVELVVRIGDMISDMADD- - - - - GPRNRVIRRNLLRCVLN DMaV QWMMKNHLNLPCKVGLNVFGFGWDEMKFKHGAYSHH-FCGDYSGFDSNTNVAMLDMVADMISDFARD- . - . - - - GARNRLIRRNLMHAAVT BRNV QFIMRMHNVLPCKVGLNPFSQDWDEMHAEHTRFEHH-FNGDYSGFDTGTPRQLLLKFADLISELAAD- - - - - GRRNKVIRRNLMQLAVD SMOV QFIMSLHDFLPCKVGLNVYSKSWDTMHAEHNRFAYH-FNGDYTGFDTATPRVLMMKIADMVSNLAGD- - - - - - - GRENAIVRRNLMKMAVE

$$
::^{*}: *^{*}: \quad *^{*}: *^{*}: *^{*} \quad . \quad \text { : }
$$

BnRV1 RDGIAGAHLLRYSQGMPSGFSMTVIFNSFVNYYYMALAWMYIVSRSELSPQADLGSFDRFTKIIV- -YGDD BVCV RDGLAGDVLLRYSQGMPSGFSMTVIFNSFVNYYYMALSWINIIANSPLSPQADLVSFDYYTKIVV- -YGDD RTSV RDGICGDLILRYSQGMPSGFAMTVIFNSFVNYYFMALAWMSTVGSSLLSPQGSCKDFDTYCKIVA- -YGDD MCDV REGIVKEYLFQYCQGMPSGFAMTVIFNSFVNYYYLAMAWMNLISHSPLSPQSTVRDFDNYCKVVV- -YGDD CNDV RFSLVKGGVLRISGGGPSGFPMTVIFNSFINLFYLQSAWIMLASQNGRSDISCPSNFPSFVRACV - YGDD PYFV RFSLMKGAILRVPGGGPSGFPMTVIFNSFINLFYLQSAWIMLARFNGRQDISHPCNFPKYVRACV - YGDD CaTV1 HYTLCGDKLFRVVGGMPSGFSLTVLFNSLLNEFYMRYAFEILLRKPANAARTIGMSQTNFNELFVAIYGDD MYMoV HFTLCEDKCYRVVGGMPSGFSLTVLFNSLLNEFYMRYAFEMLLRKPQNRARTLGMTQRNFEDLFVAIYGDD LNLCV HLTLCGDKLFRVVGGMPSGFSLTVLFNSLLNEFYMRYAFERLLHHPRNITRTMGMTQRTFSELFIAIYGDD ToMarV HYTLCEDKVFRLVGGMPSGFALTVIFNSLLNEFYMRYAFISLLRRPHIAARAIGVKPSDFNQLFIAVYGDD TOTV HYTICENKVFRLVGGMPSGFALTVIFNSLLNEFYMRYAFISLLRRPHIAAQAIGCKPSDFNKLFVAVYGDD SCLSV HLTLCGDKLFSVKGGMPSGFSLTVIFNSLLNEFYMRYAFGMLLLRSDIKARSIGVTMNDFDRIFIAVYGDD ALSV RYGLTNGDLWYVTSGIPSGFPLTVIVNSLVNSFFVHFAYMKLHSSEVHKALYPLYSFRTLVSYAV--YGDD CRLV RYGLTNGDLWYVTSGIPSGFPLTVIVNSLVNNFFIHFSYIKLMKREELNSLYPLHSFRQMVAYAT - YGDD CULV RYGLTQGDLWYVNSGIPSGFPLTVIVNSLVNSFFIHYAYMSICKKSNDMDLYPLYSFKOLVSYAV- -YGDD AVB RYGIAGLGVYKVSSGIPSGFPLTVIVNSLVNSFFLHFAYRKLMSQSLKSHFW----FTKNVAFAV- -YGDD SLRSV RLGVAGIGLYRVSGGIPPGFALTVIVNSLVNHFLVRWSWEHMMASSSLF-..--.--FTDCVELAV- -VGDD SDV RYLISDGVLYEIHGGTPSGFAPTVMINSVVNEFYLKWSWIGLLKEAGYANQATLYAFHEATEISL - - YGDD CLVA RQVIVGRDLYKVKGGTPSGFALTVMINSVVNEIYLKMAWFGLSRK-YDPLLARDADLRHHVCMSV- -YGDD DMaV RRMIVGRNIYKIIGGTPSGFALTVMVNSIINELYLKMAWFNLSKQ-HFPEISRDADLSHNVHIST - -YGDD BRNV RRILVLAELYHVRGGTPSGFALTVIINSMVNQFYLMWAWRKIMSR-ISPSMVTYRVMRTHCTFSV - YGDD SMOV RRILVLRDLYQVKGGTPSGFALTVIINSVVNQFYLMWAWRKIMSR-IDPGLVPYRVMRSHCTFSV--YGDD $: \quad: \quad * * . * * . * *: .^{* *} .:^{*} \quad: \quad:: \quad * * *$ 
Fig. S3. Multiple sequence alignments of the waikavirus ORFX proteins (a) and the overlapping region of the ORF1 polyproteins (b) Identical and similar residues in all sequences are shaded in black and gray, respectively. Note that the ORFX proteins are more well conserved than the ORF1 polyproteins.

(a)

BnRV1 1 MQR-VLLIASLAINMAALFMOVLGLLLKOPIILIVGICVIMLNIFLNVLALVTKPEEDFSOFLERASAGTPLARNAERRAPLPVRERR-- 87

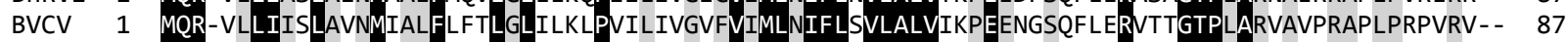

RTSV 1 MQRGFYLIICLSLSMIALFVLMLALVFRKPVLVMITLCVIMLSIFLNVLALVVRPEENFSELVARVGTGTPLARLAERRVDLPTRGRI -- 88

MCDV 1 MQR-WLLIIGLAVNMLSCFFQMLGLVFRLPLLLVIGLCVTMLNIFLSVLALVTRSEDTLLDLMTRMHIGTPRAQNVAPVARERTLGPRSR 89

(b)

BnRV1 99 AKSAINCKLGYKY-GCALYAGIGTSSQATNNYSDSWNMCDNAEHLFECFSLSDQTRGRLFSVPGESVGWHTTCAKCGASCSFAGPREAL-- 185

BVCV 99 AARVINNKFSSQY-DCSVSVHPGSNPEVTSHSDCWSVCNNAQHLFECVSFSDQTRGEWFPVLGKSNNWHTTCSGCGASCAFATPREGV- - 185

RTSV 99 AKGFLFDNLSIPFDDCALCPNAGTRLSQTGVSHDHFVCNYVEHLFECASFSRETGGKFFRACSEGWHWNATCTTCGASCRFANPRENI - - 186

MCDV 178 AEVVADNRVSCEY-AKLLLSNARVGVQVTPPACDWVVCNNVEHLFECFGISDAQRGHITGFNDENAYWNASCAKCGACCQGANARSAIPI 266 